\title{
Rational strain improvement for surfactin production: enhancing the yield and generating novel structures
}

\author{
Fangxiang Hu $\mathbb{P}$, Yuyue Liu and Shuang Li
}

\begin{abstract}
Surfactin, one of the most powerful microbial surfactants, is a lipopeptide-type biosurfactant which combines interesting physicochemical properties and biological activities. However, the high cost caused by its low productivity largely limits the commercial application of surfactin. Hence, many engineered bacterium have also been used to enhance surfactin biosynthesis. This review briefly summarizes the mechanism of surfactin biosynthesis, highlighting the synthesis pathway of $\mathrm{N}$-terminally attached fatty acids, and outlines the main genetic engineering strategies for improving the yield and generating novel structures of surfactin, including promoter engineering, enhancing efflux systems, modifying the transcriptional regulatory genes of surfactin synthase (srfA), genomics and transcriptomics analysis, non ribosomal peptide synthetase (NRPS) domain and combinatorial biosynthesis. Finally, we discuss the future prospects of the research on surfactin.
\end{abstract}

Keywords: Surfactin, Branched chain fatty acids, Biosynthesis, Structure, NRPS

\section{Background}

Since green chemicals and industrial processes have become a concern for the whole society, environmentally-friendly, biodegradable biosurfactants with low toxicity have aroused great interest $[1,2]$. Biosurfactants are classified into glycolipids, phospholipids, fatty acid lipopeptides, lipoproteins, polymeric surfactants and particulate surfactants based on their natural chemical structure and microbial origin [3, 4]. Lipopeptides constitute a class of antimicrobials composed of a hydrophilic peptide ring and hydrophobic fatty acid moieties $[5,6]$. According to their structural characteristics, lipopeptides can be divided into cyclic lipopeptides (CLPs) and linear lipopeptides [7, 8]. The cyclic lipopeptides that have been discovered so far, including fengycin, iturin and surfactin, are mainly produced by Bacillus subtilis $[9,10]$. These lipopeptides all have a peptide ring of 7 or 10 amino acids with a long hydrophobic fatty acid chain. The fatty acid

*Correspondence: lishuang@njtech.edu.cn

College of Biotechnology and Pharmaceutical Engineering, Nanjing Tech University, No. 30 Puzhu South Road, Nanjing, Jiangsu, China chain lengths differ, whereby that of surfactin is $\mathrm{C}_{13}-\mathrm{C}_{16}$, iturin's chain is $\mathrm{C}_{14}-\mathrm{C}_{17}$, and that of fengycin is $\mathrm{C}_{14}-\mathrm{C}_{18}$.

Surfactin, a secondary metabolite first discovered in the culture broth of B. subtilis in 1968 [11], is the most well-known lipopeptide with broad-spectrum antibacterial activity. Surfactin has a ring-shaped peptide chain containing seven amino acids, and a $\beta$-hydroxy fatty acid chain of 13-16 carbon atoms as shown in Fig. 1 [12]. Because of some differences in the length of the fatty acid chain and the types of amino acids, surfactin has many congeners or isoforms [13]. Due to its unique structure, surfactin can not only lower the surface tension of water from 72 to $27 \mathrm{mN} / \mathrm{m}$, but is also highly thermally stable and salt tolerant $[14,15]$. Thus, it has great potential in both enhanced oil recovery (EOR) and the microbial enhancement of oil recovery (MEOR) $[16,17]$. In addition, surfactin has been considered as a good candidate for use in bioremediation of contaminated soils and subsurface environments [18].

Although surfactin has potential therapeutic applications of anticancer therapy and environmental applications [19], it cannot compete with synthetic chemical surfactants due to its high cost and low yields. In order 


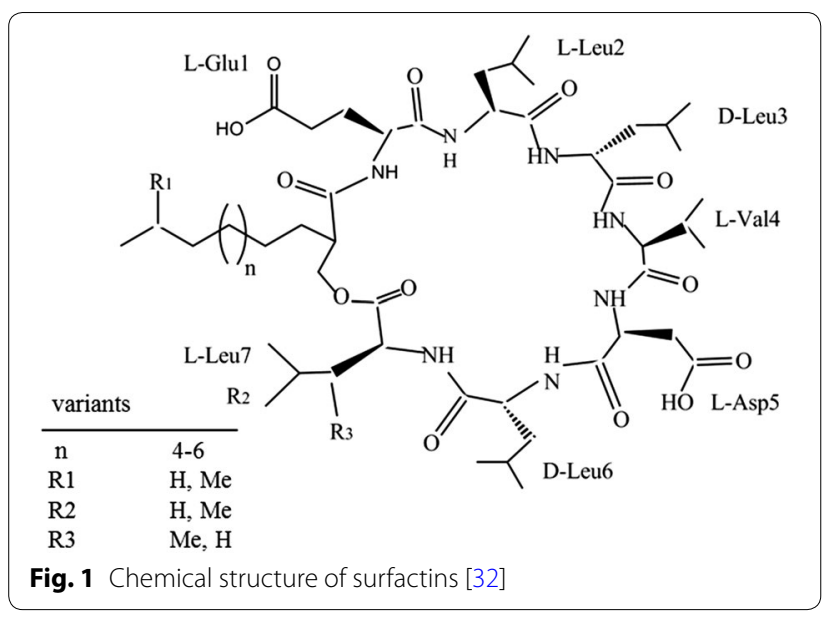

to reduce the cost, several waste materials such as feather hydrolysate waste, glutamate mill waste and distillers' grains have been tested as carbon sources for the production of surfactin, but the yields were about $500 \mathrm{mg} / \mathrm{L}$, much lower than those $(>1000 \mathrm{mg} / \mathrm{L})$ in defined medium [20-22]. In recent years, many studies on enhancing the production of surfactin have been of particular interest. Fermentation parameters including $\mathrm{pH}$, temperature, agitation speed, oxygen supply, medium composition and fermentation strategies are all important factors in surfactin production [3, 23-25]. However, with the development of genetic technology, rational engineering of strains for improved surfactin production has attracted more and more attention.

This review provides a general overview of the biosynthesis and genetic engineering strategies for enhancing surfactin production and generating novel surfactin variants. Especially, the synthetic mechanism of surfactin is not only discussed in terms of non-ribosomal peptide synthetases (NRPS); also, the biosynthesis of branchedchain fatty acids and branched-chain amino acids will be highlighted.

\section{Biosynthesis of surfactin and N-terminally attached fatty acids}

Surfactin is one of the most prominent and well-studied members of the class of lipopeptides. Surfactin is synthesized by large multifunctional NRPS that contain three modules, SrfAA, SrfAB and SrfAC, which compose a linear array of seven modules (one module per residue) with each module being responsible for the addition of one amino acid [26, 27]. Each module contains at least three catalytic domains: an adenylation domain (A) is responsible for the selection and activation of the substrate, a small peptidyl carrier protein (PCP) carries the aminoacyl-adenylate substrate as enzyme-bound thioester, and a condensation (C) domain forms the peptide bonds between the acyl-S-PCP intermediates [28, 29]. Epimerization (E) domains perform the stereochemical conversion to yield the D-isomer of some of the incorporated residues. An additional thioesterase (TE) of the termination module catalyzes product release by either hydrolysis or macrocyclization, to generate either cyclic or cyclic-branched molecules [30,31].

The synthesis process of surfactin can be separated into three parts, the initiation of synthesis, the elongation of the peptide chain and the cyclization of the peptide chain. The first module $\left(\mathrm{C}-\mathrm{A}_{\mathrm{Glu}}-\mathrm{PCP}\right)$ of surfactin synthetase SrfAA is responsible for the lipoinitiation reaction [32]. The donor site of the starter $C$ domain has a very distinct specificity for the chain length of its 3-hydroxy fatty acid substrate.

Although the N-terminally attached fatty acids are key structural elements of surfactin, few researchers paid attention to their biosynthesis pathway [33-35]. Among the produced surfactin variants, those with branched-chain fatty acids are the main component, accounting for about $78 \%$ of the total [27]. Hence, the fatty acid biosynthesis system, especially that for branched-chain fatty acids, is also critical for the synthesis of surfactin in addition to NRPS. Beta-ketoacyl-acyl carrier protein synthase III $(\mathrm{FabH})$ catalyzes the condensation of malonyl-acyl carrier protein (ACP) with acetyl-CoA to form $\beta$-ketobutyryl-ACP, which is the initial step of straight chain saturated fatty acid biosynthesis. However, the FabH of B. subtilis can initiate the straight- and branched-chain fatty acid synthesis cycle by condensing acetyl-CoA, isobutyryl-CoA, isovalerylCoA or $\alpha$-methylbutyryl-CoA with malonyl-ACP, and it showed higher activity and selectivity for branched-chain fatty acid synthesis precursors [36, 37]. In Bacillus, the branched-chain fatty acid phospholipids are the main components of cell membrane phospholipids, accounting for $96 \%$ of total phospholipids (iso-C14: 0, 4\%, iso-C15: 0, 24\%, iso-C16: $0,12 \%$, iso-C17: $0,13 \%$, anteiso-C15: 0 , 34\%, anteiso-C17: 0, 9\%, normal-C16: 0, 4\%) [38]. The branched-chain fatty acid synthesis precursors isobutyryl-CoA, isovaleryl-CoA and $\alpha$-methylbutyryl-CoA can be derived from the branched-chain amino acids L-valine, L-leucine and L-isoleucine, respectively. Thus, the biosynthesis of branched-chain amino acids and branched-chain fatty acids also greatly influences surfactin biosynthesis.

As shown in Fig. 2, the process by which the branched chain amino acids L-isoleucine, L-valine and L-leucine participate in surfactin biosynthesis was divided into three parts: branched-chain amino acids biosynthesis, branched chain fatty acid and CoA-activated 3-hydroxy fatty acids precursor biosynthesis, and NRPS-catalyzed 


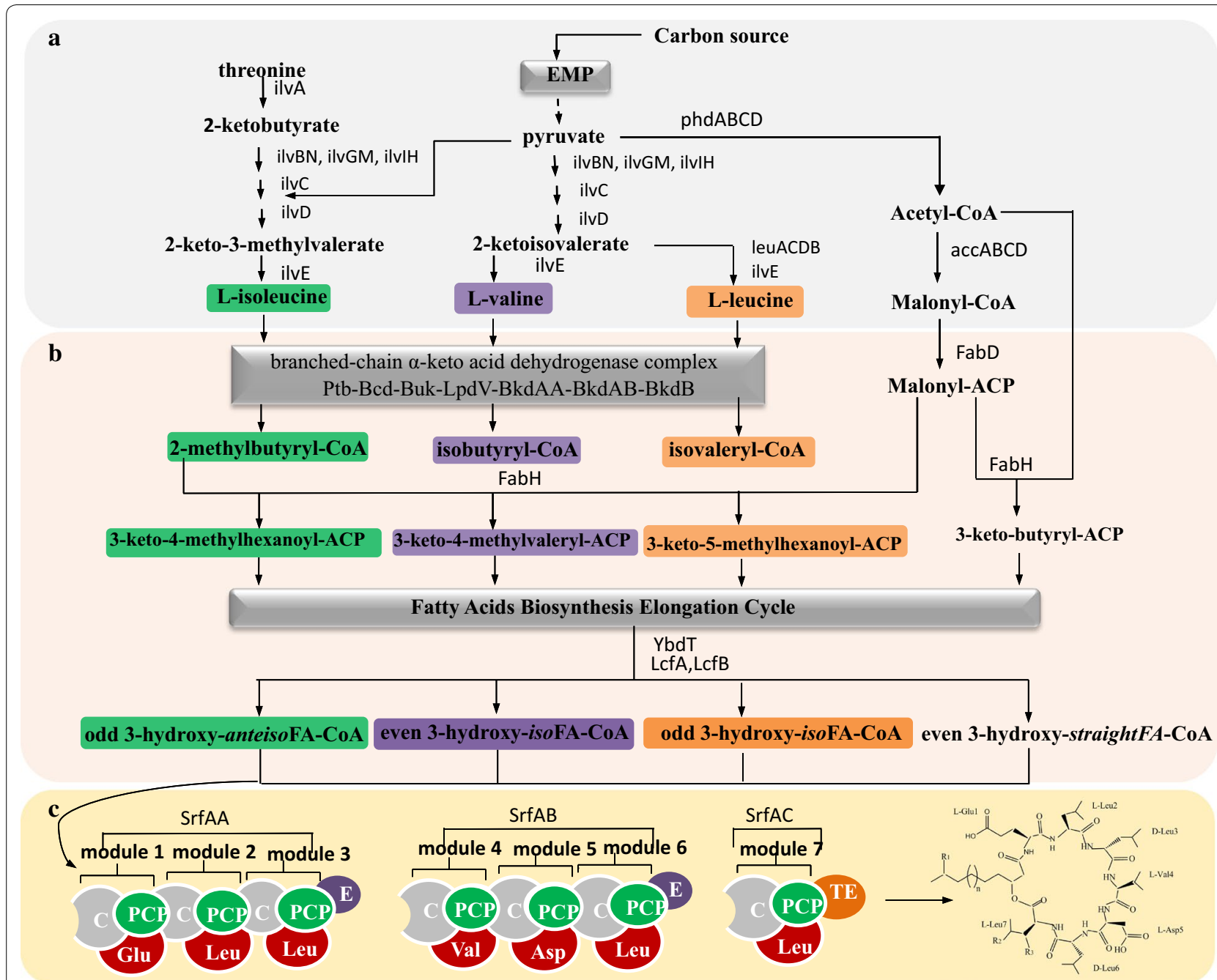

Fig. 2 The biosynthesis pathways of branched-chain amino acids participating in surfactin biosynthesis. a Branched-chain amino acid biosynthesis module, represented by light grey panel. ilvA, L-threonine dehydratase; ilvBN, acetohydroxy acid synthase I; ilvGM, acetohydroxy acid synthase II; ilvIH, acetohydroxy acid synthase III; ilvC, acetohydroxy acid isomeroreductase; ilvD, dihydroxy acid dehydratase; leuACDB: leuA, 2-isopropylmate synthase; leuCD, isopropylmalate isomerase; leuB, 3-isopropylmalate dehydrogenase; EMP, Embden-Meyerhof-Parnas pathway, marked with deep gray panel; ilvE, branched chain amino acid aminotransferase; phdABCD, pyruvate dehydrogenase; accABCD, acetyl-CoA carboxylase. b Biosynthesis of branched-chain fatty acids and CoA-activated 3-hydroxy long chain fatty acids, represented by light orange panel. fabD, malonyl-CoA:ACP transacylase; FabH, $\beta$-ketoacyl-ACP synthases; Branched-chain a-keto acid dehydrogenase complex marked with deep gray panel; Ptb, butyryl coenzyme A transferase; Bcd, L-leucine dehydrogenase; Buk, butyrate kinase; LpdV, 2-oxoisovalerate dehydrogenase; BkdAA, 2-oxoisovalerate dehydrogenase; $B k d A B$, 2-oxoisovalerate dehydrogenase; BkdB, 2-oxoisovalerate dehydrogenase; YbdT, fatty acid beta-hydroxylating cytochrome P450 enzyme; LCFA and LcfB, long-chain fatty acid-CoA ligases. FAB, fatty acid biosynthesis. The degradation pathway of L-isoleucine was marked with green panels; the degradation pathway of $L$-valine was marked with purple panels; the degradation pathway of L-leucine was marked with orange panels. c Nonribosomal peptide synthetase synthesis module. A, adenylation domain, represented by amino acids in red colour; PCP, peptidyl carrier protein domains, shown in green colour; $C$, condensation domain, shown in gray colour; E, epimerization domain, shown in purple colour; TE, thioesterase domain, shown in orange colour

synthesis. The biosynthesis of the branched-chain amino acids L-isoleucine, L-valine and L-leucine shares the same enzyme system encoded by ilvBN, ilvGM, ilvIH, ilvC, ilvD, and ilvE $[39,40]$. Especially, the production of L-leucine from the intermediate precursor $\alpha$-ketoisovalerate involves an enzyme complex encoded by leuACDB. The resulting intermediates are converted into the corresponding branched-chain acyl-CoA precursors: $\alpha$-methylbutyryl-CoA, isobutyryl-CoA, and isovaleryl$\mathrm{CoA}$ through the branched-chain $\alpha$-keto acid dehydrogenase complex [41]. Subsequently, these branched-chain acyl-CoAs and malonyl-ACP are condensed to yield 3-keto-4-methylhexanoyl-ACP, 3-keto-4-methylvaleryl$\mathrm{ACP}$ and 3-keto-5-methylhexanoyl-ACP by the action 
of FabH [42]. At the same time, acetyl-CoA is condensed into 3-keto-butyryl-ACP which is the precursor of straight chain fatty acids. Then, these fatty acyl precursors enter into the fatty acid biosynthesis elongation cycle to produce corresponding branched- and straight-chain fatty acids, as shown in Fig. 2 [43]. Next, the respective fatty acids are hydroxylated by the cytochrome P450 enzyme YbdT, which was proved to be responsible for the 3-hydroxylation of long chain fatty acids (LCFAs) $[44,45]$. The subsequent activation of 3-hydroxy LCFAs occurs via the activity of acyl-CoA ligases LcfA and LcfB in B. subtilis [32]. The final CoA-activated LCFAs are recognized as substrates for the initiation of surfactin synthesis.

The importance of branched-chain amino acid and branched-chain fatty acid synthesis was recently confirmed by transcriptome analysis. The genome and transcriptome analysis of B. amyloliquefaciens MT45, a high-producing strain with a surfactin titer of $2.93 \mathrm{~g} / \mathrm{L}$, showed that most genes in fatty acid synthesis pathway were upregulated compared with $B$. amyloliquefaciens type strain DSM7T, which produced much less surfactin [46]. Furthermore, genes participating in acetyl-CoA generation, which is a precursor of fatty acids, were also particularly upregulated. Likewise, the results of our recent work showed that Bacillus velezensis BS-37 [47], a surfactin high-yield strain, were in agreement with the transcriptome analysis. Moreover, the surfactin production of strain BS-37 doubled to nearly $2 \mathrm{~g} / \mathrm{L}$ with the addition of $10 \mathrm{mM} \mathrm{L}$-Leu, which was consistent with a previous report that surfactin production could be increased 20.9-fold by strengthening the leucine metabolic pathway [35]. In addition to these findings, there were many studies that proved the importance of branched-chain amino acids and branched-chain fatty acids. For example, the deletion of the gene $l p d V$, encoding a part of the dehydrogenase complex responsible for the conversion of CoA-precursors to respective branched chain FAs, causing surfactin C14 isoform with straight FA chain was twofold more than the wild type. The research of Kraas et al. showed that the deletion of LcfA and LcfB, which were proposed to activate fatty acids for degradation, causing the production of surfactin to decrease by $84 \%$ [32].

\section{Strategies for enhancing surfactin production}

Most reported surfactin titers of wild-type bacterial producers are in the range of $100-600 \mathrm{mg} / \mathrm{L}$. For example, B. subtilis BS-37 was reported to produce $585 \mathrm{mg} / \mathrm{L}$ [16], and B. velezensis H3 $488 \mathrm{mg} / \mathrm{L}$ [48]. It has been difficult to achieve significant breakthroughs in production only through traditional strategies of mutagenesis and breeding or fermentation optimization. Consequently, establishing genetically modified surfactin producer strains is of great significance. In terms of strain modification to improve the production of surfactin, there are mainly three strategies: (1) substituting the native promoters $\mathrm{P}_{\text {srf }}$ of $s r f A$ modules, which is important for surfactin synthesis; (2) strengthening the efflux of surfactin by overexpression of assistant proteins and surfactin transporters is also an effective way. (3) To modify the transcriptional regulatory genes of $\operatorname{srfA}$.

\section{Promoter engineering}

The production of surfactin requires the critical $\operatorname{srf} A B$ locus, which is a large operon of $27 \mathrm{~kb}$ controlled by the promoter $\mathrm{P}_{\text {srf }}$. Because it is difficult to heterologously express $s r f A$, promoter exchange has been regarded as a preferred way to improve the productivity of surfactin. There are three types of promoters often used in B. subtilis: inducer-specific promoters, constitutive promoters and autoinducible promoters [49]. The best-known constitutive promoter is $P_{43}$. The inducible promoter $\mathrm{P}_{\mathrm{xyl}}$ is driven by xylose, $\mathrm{P}_{\mathrm{spac}}$ is induced by IPTG. Auto-inducible promoters (e.g., $\mathrm{P}_{\text {pst }}$ and $\mathrm{P}_{\text {cry3Aa }}$ ) can be used to express the target gene from the late log phase to the stationary phase [50]. $\mathrm{P}_{\text {srf }}$ is an autoinducible promoter which is triggered by signal molecules acting in a quorum sensing pathway. The recent successes in promoter exchange to promote the yield of surfactin were achieved using natural high-yield strains $[51,52]$. In an earlier study, Sun et al. used the IPTG-inducible hybrid promoter $\mathrm{P}_{\text {spac }}$ to replace the native $\mathrm{P}_{\text {srf }}$ promoter of $B$. subtilis $\mathrm{fmbR}$, which resulted in a tenfold surfactin yield enhancement, to about $3.86 \mathrm{~g} / \mathrm{L}$ [53]. In addition to using natural strong promoters, synthetic promoters were also investigated, and perhaps the most remarkable result was achieved by Song et al. [51]. They identified several strong native promoters $\left(\mathrm{P}_{\mathrm{groE}}, \mathrm{P}_{\mathrm{sacB}}\right.$ and $\left.\mathrm{P}_{\mathrm{sacP}}\right)$ in B. subtilis THY-7 through transcriptome analysis and confirmed the weakness of the native $\operatorname{srfA}$ promoter. However, the recombinant strains could not successfully synthesize surfactin using the strong constitutive $\mathrm{P}_{\text {groE }}$ core promoter. Afterwards, three novel promoters were designed using the $\mathrm{P}_{\text {groE }}$ core promoter as the basis. When the ultra-strong chimeric promoter $\mathrm{P}_{\mathrm{g} 3}$ was used to drive surfactin synthesis, the surfactin titer in flasks reached $8.61 \mathrm{~g} / \mathrm{L}$, which was 15.6 -fold greater than that of wild-type THY-7.

However, there are also reports of failures of promoter modification in model strains or other organisms. Coutte et al. reported that the surfactin concentration changed from 1.5 to $1.2 \mathrm{~g} / \mathrm{L}$ after replacing the $\mathrm{P}_{\text {srfa }}$ of the $B$. subtilis 168-derived strain 
BBG111 with the constitutive promoter $\mathrm{P}_{\text {repu }}$ originating from the replication gene repU of the Staphylococcus aureus plasmid pUB110 [54]. Subsequently, Willenbacher et al. analyzed the effect of promoter replacement in minor and strong surfactin producer strains. They found the surfactin concentration of minor producer strains $B$. subtilis 3A38 was increased, while the strong producer strains $B$. subtilis DSM $10 \mathrm{~T}$ was decreased after substitution of the native promoter using constitutive promoter $\mathrm{P}_{\mathrm{veg}}$, as shown in Table 1 [52].

Overall, to establish genetically modified high-yield surfactin producer strains by promoter engineering, it is necessary to analyze the transcriptome and production capacity of the strain. Targeted construction of a strong promoter or hybrid promoter suitable for each strain may be more suitable. However, it is also necessary to consider that there are many uncertainties in the genetic operating system of wild-type strains, which greatly increases the difficulty of genetic manipulation.

\section{Enhancement of the efflux of surfactin}

The mechanism guiding the transmembrane efflux of surfactin is not very clear, but surfactin has a deep effect on the lipids of biological membranes [55]. As reported, the surfactin monomer can insert itself into phospholipid layers in biomimetic membrane systems at low concentrations (below or near the critical micelle concentration, $\mathrm{CMC}$ ). At higher concentrations, surfactin can cause membrane solubilization and vesicle destruction [56]. Some researchers speculated that the transmembrane efflux of surfactin may simply be the result of membrane insertion and penetration of surfactin monomers or oligomers [57]. In an early report, Tsuge et al. [58] suggested that there may be a mechanism for surfactin efflux mediated by protein transporters. They found that the production and resistance to surfactin of B. subtilis hosts could be significantly reduced by null mutations in yerp, a gene encoding a protein with homology to RND (resistance, nodulation and cell division) family efflux pumps. The speculation that transmembrane exporters dependent on the proton motive force (PMF) could facilitate the efflux of surfactin in $B$. subtilis was further confirmed by $\mathrm{Li}$ et al. According to the energy source, bacterial transmembrane transporters can be divided into two categories, those dependent on ATP or proton motive force (PMF, i.e. transmembrane proton concentration gradient) [59]. Firstly, Li et al. used liposomes and transmembrane transport inhibitors to confirm that the surfactin efflux in THY-7 was mainly dependent on the PMF, and not ATP hydrolysis. Then, they identified the putative lipopeptide transporter YcxA, which depends on the PMF, unable to transfer surfactin in THY-7 due to a frameshift mutation of the encoding gene. Afterwards, three putative lipopeptide transporters with PMF as energy source were overexpressed, and the

Table 1 Surfactin yields of recombinant strains

\begin{tabular}{|c|c|c|c|}
\hline Strain & Description & Production $(g / L)$ & References \\
\hline B. subtilis fmbR & Native $P_{\text {srfa }}$ & 0.38 & [53] \\
\hline B. subtilis fmbR-1 & Replacement of $\mathrm{P}_{\text {srfa }}$ with $\mathrm{P}_{\text {spac }}$ & 3.86 & \\
\hline B. subtilis THY-7 & Native $P_{\text {srfa }}$ & 0.55 & [51] \\
\hline B. subtilis THY-7/Pg3-srfA & Replacement of $\mathrm{P}_{\text {srfA }}$ with $\mathrm{P}_{\mathrm{g} 3}$ & 9.74 & \\
\hline B. subtilis BBG111 & Native $P_{\text {srfa }}$ & 1.5 & [54] \\
\hline B. subtilis BBG113 & Replacement of $\mathrm{P}_{\text {srfa }}$ with $\mathrm{P}_{\text {repU }}$ & 1.2 & \\
\hline B. subtilis 3A3B & Native $P_{\text {srfa }}$ & 0.07 & [52] \\
\hline B. subtilis JWSurf2 & Replacement of $P_{\text {srfA }}$ with $P_{\text {veg }}$ & 0.26 & \\
\hline B. subtilis DSM 10T & Native $P_{\text {srf }}$ & 0.62 & [52] \\
\hline B. subtilis JWSurf3 & Replacement of $\mathrm{P}_{\text {srfA }}$ with $\mathrm{P}_{\text {veg }}$ & 0.04 & \\
\hline B. subtilis THY-7 & - & 0.55 & [57] \\
\hline B. subtilis TS589 & Overexpression of THY-7-P grac $-y c X A$ & 1.15 & \\
\hline B. subtilis TS593 & Overexpression of THY-7-P grac $-k r s E$ & 0.93 & \\
\hline B. subtilis TS662 & Overexpression of THY-7-P grac $^{-y e r P}$ & 1.67 & \\
\hline B. subtilis & - & 0.021 & [69] \\
\hline B. subtilis (pHT43-comXphrC) & Overexpression of ComX and PhrC & 0.135 & \\
\hline B. subtilis BBG258 & $\begin{array}{l}\text { Insertion of the sfp gene in the amyE locus of } B \text {. } \\
\text { subtilis } 168\end{array}$ & 0.221 & [35] \\
\hline B. subtilis BBG260 & Knocking out codY in BBG258 & 2.289 & \\
\hline
\end{tabular}




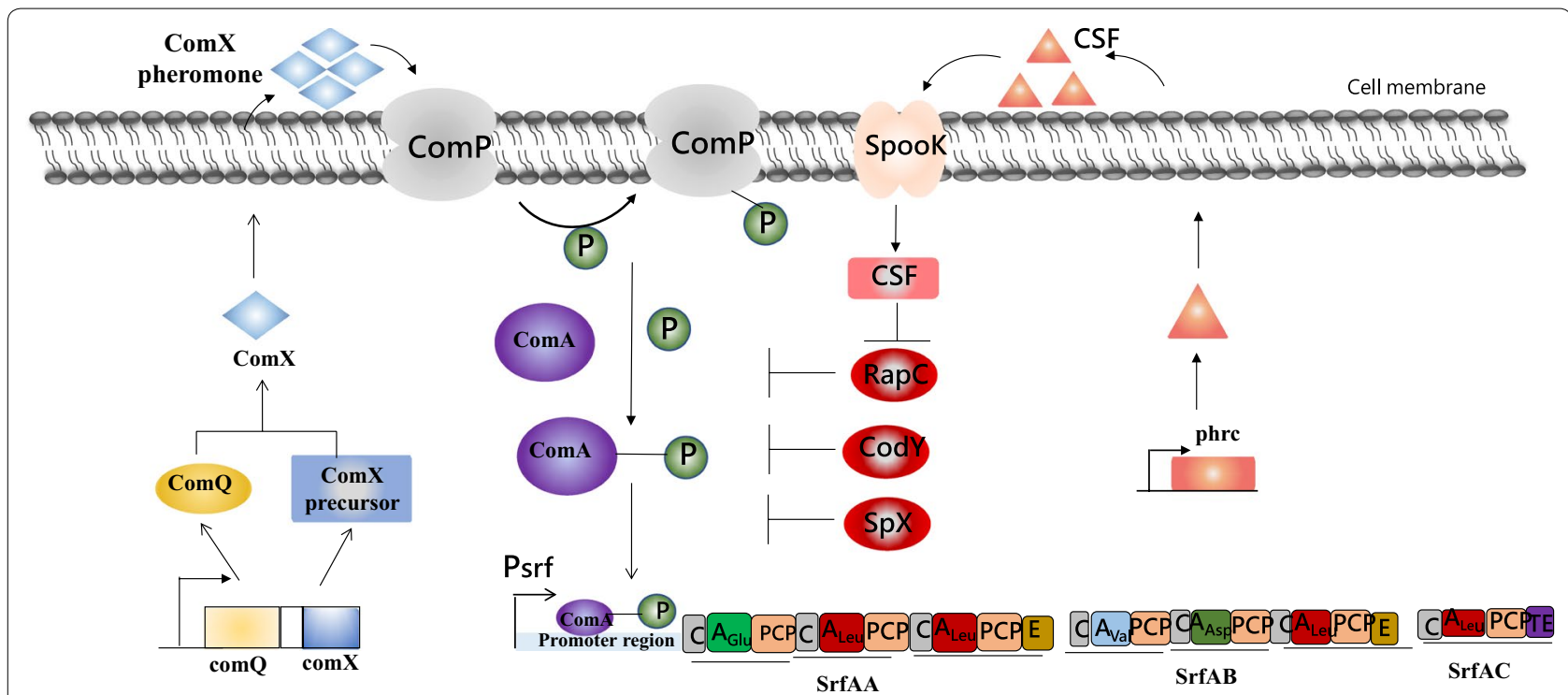

Fig. 3 The schematic model for the regulation of the transcription of the srfA operon network involved in two extracellular signaling peptide-mediated quorum sensing in B. subtilis. T-bars indicate the negative effects on DNA binding or protein interactions. Bent arrow represents the promoter. 'P' in the circle represents the phosphoryl group

secretion of surfactin increased by $89 \%, 52 \%$ and $145 \%$ with the overexpression of the natural full-length YcxA, KrsE and YerP proteins.

To enhance the efflux of surfactin, proteomics can be used to analyze the differences between key proteins related to efflux in different host strains, especially wildtype strains with high productivity of surfactin. However, the ability of surfactin efflux in the strain needs to be identified first. The mechanism of surfactin efflux and proteins involved in transmembrane transport still need to be further researched.

\section{Modifying the transcriptional regulatory genes of $s r f A$ operon}

The expression of $\operatorname{srfA}$ operon is not only controlled by the promotor $\mathrm{P}_{\text {srf }}$, but also can be regulated by some transcriptional regulatory genes. Bacterial quorum sensing (QS) system, a cell-density-dependent regulatory networks, plays a significant role in the regulation of srfA operon. The two canonical peptides, ComX and CSF, mediate the quorum sensing control of competence and sporulation in B. subtilis [60,61]. In the late-growth phase, a series of gene transcription events downstream of the ComA-ComP two-component are triggered by the accumulation of a high concentration of peptides, among which the $\operatorname{srfA}$ operon is stimulated via a complex-regulation phosphorylated cascade [62-64]. As shown in Fig. 3, both ComX and CSF activate the transcription of the $\operatorname{srfA}$ operon by stimulating the activity of transcription factor ComA through phosphorylation (ComA-P) via two separate pathways. In one pathway, the monitor of cell density, the ComX pheromone, is firstly modified by the ComQ isoprenyl transferase to be processed outside. The signal transduction system composed of the two-component regulatory proteins ComP and ComA is activated when the ComX pheromone reaches the obligatory concentration. Ultimately, phosphorylated ComA binds to the promoter $\left(\mathrm{P}_{\text {srfA }}\right)$ of the srfA operon in tetramer form, and cooperates with SigA to activate the transcription of corresponding gene expression [65]. Another pathway is mediated by CSF, which is encoded by $p h r C$ and is initially imported as inactive-form CSF (inact). CSF is imported into the cell by the oligopeptide permease SpooK [66], and then binds to the Rap protein, which causes the Rap protein losing phosphatase activity. Thereby, the dephosphorylation of phosphorylated ComA can be prevented to facilitate the transcription of srfA gene and synthesis of surfactin [67].

In early research, the expression of $s r f A$ was decreased by inhibiting the transcription of the ComQXP quorumsensing locus in B. subtilis [68]. In addition, Wang et al. found that surfactin production was severely decreased by a mutation of three non-aspartate amino acids in the ComA response regulator receiver motif. Later, Jung et al. confirmed that the overexpression of signaling factor ComX and PhrC could successfully increase the production of surfactin [69].

In addition to the ComX and CSF, the expression of the $\operatorname{srfA}$ operon is also regulated through several global regulators and regulatory protein. The global regulator $\operatorname{Cod} Y$ 
can repress $s r f A$ transcription through directly binding to the $\operatorname{srfA}$ promoter regions $[70,71]$. The $\operatorname{cod} Y$-dependent repression of $\operatorname{srfA}$ transcription can be triggered by high external concentrations of amino acids. Consequently, the surfactin production in B. subtilis 168 derived strains was increased about tenfold through the knockout of $\operatorname{cod} Y$ [35]. The regulatory protein $\mathrm{SpX}$ suppresses $\operatorname{srfA}$ expression by blocking the interaction between ComA and RNA polymerase (RNAP) in the promoter region via competition for an overlapping site in the $\alpha$-CTD [72]. Some other negative regulators of $\operatorname{srfA}$ was also detailly reported such as PerR, SinI and PhoP [33, 46, 73, 74].

The synthesis of surfactin is closely related to QS, and ComX seems to be a key factor in enhanced surfactin productivity. However, not much is known about the dynamics of the regulatory network or overall synthesized quantities. At present, the surfactin productivity of strains used in studies on the synthesis of QS regulatory genes is generally low. Thus, whether these QS regulatory genes and their regulation in high-yield strains have changed, as well as if there exist other signal peptides like $\mathrm{PhrC}$, awaits further study.

\section{The genomics and transcriptomics analysis assisted rational strain improvement}

Genome sequencing in combination with global transcriptome analysis is an effective strategy to unravel the biosynthesis and regulatory features of surfactin exhibited in high-producing strains; They can provide fundamental information for rational strain improvement via genetic modification or pathway engineering. The general regulatory network of surfactin synthesis could be divided into three functional modules: precursors supply module, intermediary transcriptional driving module and efflux and resistance module, as shown in Fig. 4.

Compared to low-producing strain Bacillus amyloliquefaciens $\mathrm{DSM}^{\mathrm{T}}$ [75], the up-regulated expressed genes in high-producing strain B. amyloliquefaciens MT45 mainly be involved in srfA operon expression, precursor redirection, and antibiotic resistance capacity. For example, the $\operatorname{srfA}$ operon was up-regulated about 9.25-48.86-fold in high-producing strain B. amyloliquefaciens MT45 [46]. Apart from this, most genes in the fatty acid synthesis pathway, such as $f a b G$, $f a \mathrm{bZ}, f a b I$ and $f a b F$ were also up-regulated. Moreover, in efflux and resistance module, $s w r C$ (synonymous of yerP) and other two genes $a c r B$ and liaRSFGHI operon were all highly expressed. AcrB had high amino acid sequence similarity to SwrC $(\sim 41 \%)$ and liaRSFGHI operon was annotated as genes associated with the resistance of daptomycin (a structural analogue of surfactin). Meanwhile, the $\operatorname{srfA}$ operon and $s w r C$ were also highly expressed in high-yield strain Bacillus velezensis BS-37 [47].
Most recently, Wu et al. [33] used B. subtilis 168 as the initial host to construct a surfactin hyperproducer through a systematic metabolic engineering method based on the results of genomics and transcriptomics of Bacillus amyloliquefaciens MT45. Three functional modules in B. subtilis 168 were strengthened via more than 40 steps of manipulations; the final surfactin titer reached up to $12.8 \mathrm{~g} / \mathrm{L}$. The surfactin production of this functional strain is also the highest yield reported so far. Compared with the wild strain B. subtilis THY-7 derived high producer recombinants $(9.74 \mathrm{~g} / \mathrm{L})$, we can speculate that the wild high producers could be more efficient for constructing a surfactin hyperproducer for industrial application, if efficient genetic manipulations were available.

\section{Structural modification of surfactins}

In addition to the use of genetically engineered surfactin-producing strains to enhance the production, some strategies were developed to modify the structures of surfactins. Combinatorial biosynthesis, a genetical engineering technique that purposefully alters the biosynthetic pathway of natural products to form predictable new structural products, plays an important role in the structural modification of cyclolipopeptide antibiotics [76]. These novel structural compounds exhibit new functions or activities as expected by the investigator. The strategies for structural modifications are mainly focused on the peptide ring and hydrophobic fatty acid chain.

\section{Surfactin variants with novel peptides}

The peptide ring of seven amino acids in surfactin is synthesized by NRPS modules. The site-directed mutagenesis, substitution, insertion, deletion, and reprogramming of peptide modules within a NRPS biosynthetic template can provide an almost infinite recombination potential to generate novel peptides. Under these circumstances, various approaches have been tested to rationally design novel surfactin products, as shown in Table 2. Some modifications were mainly focused on the substrate specificity of NRPS. Previously, the modification of surfactin was conducted by rational module swapping, such as the targeted replacement of the simplest peptide synthetase module SrfA-C [77, 78]. Later, Stachelhaus et al. extended this method to exchange the leucine-activating module within the multi-modular SrfA-A [79]. As a result, ornithine was directly incorporated at the second position of the peptide chain. However, the substitution caused a more substantial modification in the conformation of the peptide product. For example, through structural dissection of the phenylalanine-activating adenylation (A) domain, which is responsible for the specific recognition of the cognate substrate amino acid in nonribosomal 


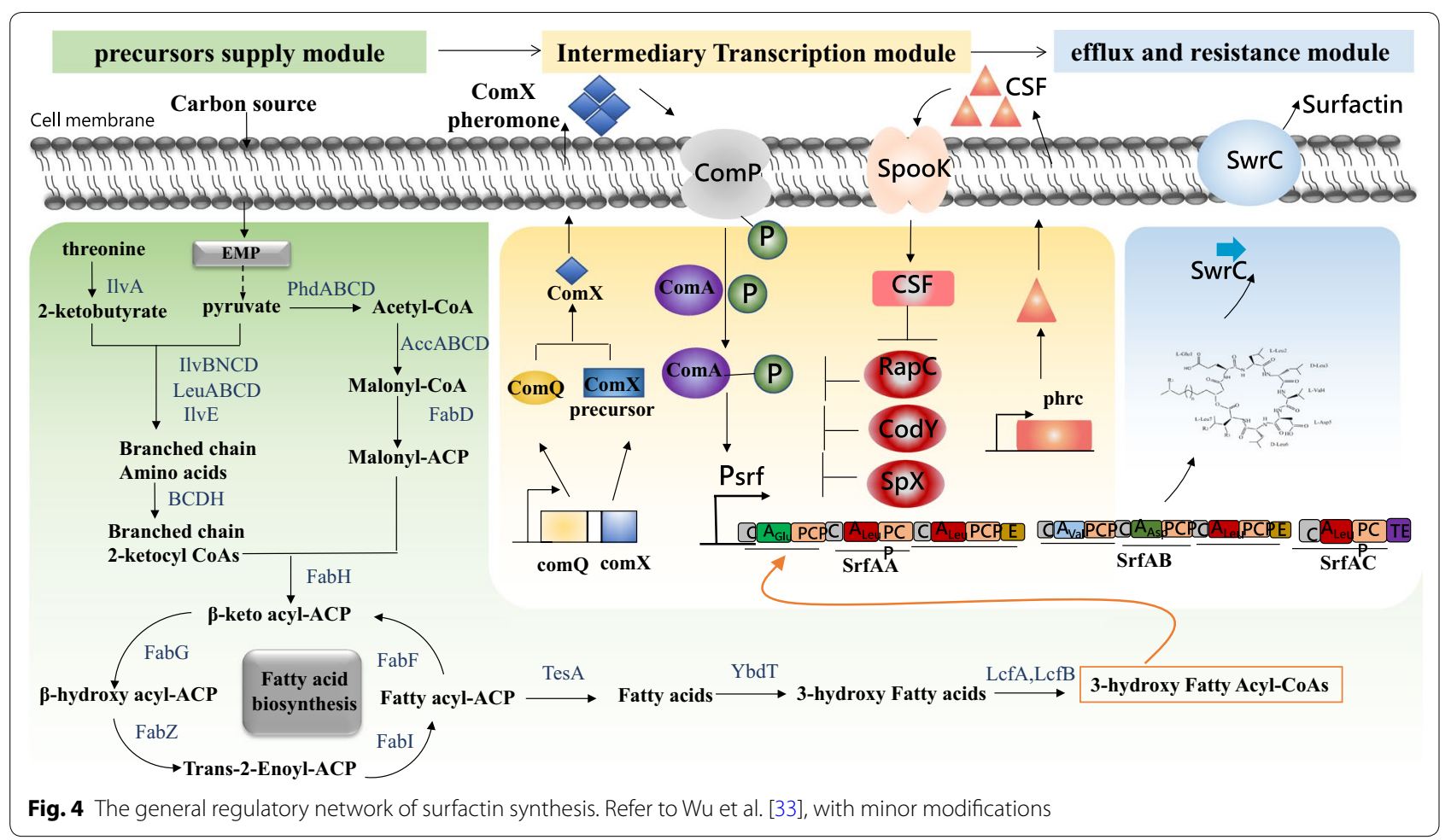

Table 2 Novel surfactin variants with modifications of the peptide ring

\begin{tabular}{|c|c|c|c|c|c|}
\hline Strain & Modification & Variants & Yields (mg/L) & Properties & Reference \\
\hline B. subtilis ATCC2133 & Substitution & {$\left[\mathrm{Cys}^{7}\right]$} & - & Decreased hemolytic activity & [77] \\
\hline B. subtilis ATCC21332 & Substitution and deletion & $\begin{array}{l}\text { 1. }\left[\Delta\left(\mathrm{Val}^{4}{ }^{4}-\mathrm{Leu}^{3}\right) \mathrm{Orn}^{2}\right] \\
\text { 2. }\left[\Delta\left(\mathrm{Val}^{4}\right) \mathrm{Orn}^{2}\right] \\
\text { 3. }\left[\Delta\left(\mathrm{Leu}^{7}-\mathrm{Leu}^{6}\right) \mathrm{Orn}^{2}\right] \\
\text { 4. }\left[\Delta\left(\mathrm{Leu}^{7}\right) \mathrm{Orn}^{2}\right]\end{array}$ & $10-20 \%$ of original strain & Decreased hemolytic activity & [79] \\
\hline B. subtilis KE100 & Mutagenesis and substitution & $\begin{array}{l}\text { 1. }\left[G \mathrm{Gn}^{1}\right] \\
\text { 2. }\left[\mathrm{Asn}^{5}\right]\end{array}$ & - & - & {$[80]$} \\
\hline B. subtilis ATCC & Deletion & {$\left[\Delta \mathrm{Leu}^{2}\right]$} & $25-50$ & $\begin{array}{l}\text { Higher antibacterial ability and } \\
\text { decreased hemolytic activity }\end{array}$ & {$[82]$} \\
\hline B. subtilis BP2-L1 & Deletion & $\begin{array}{l}\text { 1. }\left[\Delta \mathrm{Leu}^{3}\right] \\
\text { 2. }\left[\Delta \mathrm{Leu}^{6}\right] \\
\text { 3. }\left[\Delta \mathrm{Asp}^{5}\right]\end{array}$ & $0.82-1.35$ & $\begin{array}{l}{[\Delta \text { Leu3] and }[\triangle \text { Leu6] with antifun- }} \\
\text { gal activity and reduced toxicity; } \\
{[\triangle \mathrm{Asp} 5] \text { with higher antimicro- }} \\
\text { bial activity }\end{array}$ & [83] \\
\hline B. subtilis OKB10 & Deletion & [FA-Glu] & $200-250$ & $\begin{array}{l}\text { Lower CMC and higher water } \\
\text { solubility }\end{array}$ & {$[84,85]$} \\
\hline
\end{tabular}

"-" means not mentioned

peptide synthetases (NRPSs), Eppelmann et al. rationally altered the substrate selectivity of the initiation module $\mathrm{C}-\mathrm{A}_{\mathrm{Glu}}-\mathrm{PCP}$ of the surfactin synthetase complex from $\mathrm{L}-$ Glu to L-Gln using site-directed mutagenesis [80]. What's more, a novel surfactin with an $\mathrm{L}$-Asn residue at position 5 replacing the native $\mathrm{L}$-Asp constituent was produced in the same way. Moreover, the NRPS was reprogrammed to be more "clickable" by a single tryptophan-to-serine mutation in the phenylalanine-specific NRPS adenylation domain, which enabled the efficient activation of nonnatural aromatic amino acids functionalized with azide and alkyne groups [81]. These results greatly enriched the diversity of peptide modules by "click" reactions, which represent Huisgen cyclizations between alkynes and azides. 
Decreasing the ring size by means of genetic engineering is another way to produce novel peptide products. Researchers obtained a condensed ring product by deleting the leucine-incorporating SrfA-A2 module of the surfactin NRPS, resulting in a hexapeptide, $\Delta 2$-surfactin variant with a decreased ring size [82]. The hexapeptide had reduced toxicity to erythrocytes with higher antibacterial ability. However, due to the instability of the structure, the yield of hexapeptide surfactin was only $5-10 \%$ that of the wild-type product, about $25-50 \mathrm{mg} / \mathrm{L}$. Likewise, Jiang et al. knocked out the modules SrfA-A-Leu, SrfA-B-Asp, and SrfA-B-Leu of the surfactin NRPS in $B$. subtilis BP2-L1, respectively [83]. These modifications resulted in three novel hexapeptide surfactins, individually lacking the amino acids Leu-3, Asp-5, and Leu-6. Although $\left[\Delta \mathrm{Leu}^{3}\right]$ surfactin and $\left[\Delta \mathrm{Leu}^{6}\right]$ surfactin showed reduced toxicity, and the $\left[\Delta \mathrm{Asp}^{5}\right]$ surfactin showed stronger antimicrobial activity than the native surfactin, the yields of the novel products were extremely low, only $0.82-1.35 \mathrm{mg} / \mathrm{L}$. Moreover, the size of the peptide ring could be minimized by the deletion of six amino acids, with only a single glutamic acid residue remaining. This surfactin variant, fatty-acyl-glutamate (FA-Glu) had a lower $\mathrm{CMC}$ and higher water solubility than myristoyl glutamate, a commercial surfactant. However, the yield of FA-Glu was also low, reaching only about $200-250 \mathrm{mg} / \mathrm{L}$ which was $5 \%$ of that of the wild-type product $[84,85]$.

Combinatorial biosynthesis technology presents both opportunities and challenges in developing new surfactant structures and expanding their applications. The reprogramming and deleting peptide modules of surfactin can result in many novel surfactin products with new or better capabilities, but many of the reported engineering attempts faced low product yields or even inactive hybrid enzymes. Indeed, the complexity of the NRPS structure far exceeds people's imagination. The integrity of the modules and the diversification of the link area always brings uncertainty to the genetic manipulation. The extensive unexpected modification of the peptideproduct and the low yield all need to be overcome.

\section{Modification of the fatty acyl structure of surfactin}

Compared with the NRPS-catalyzed synthesis of the peptide structure, the biosynthesis of the fatty acyl structure of the surfactin molecule still needs further research. The fatty acid chain length of surfactin is generally $\mathrm{C}_{13}-\mathrm{C}_{15}$, whereby $C_{14}$ and $C_{15}$ are usually predominant $(60-80 \%)$ [34]. The length and isomerism of the FA chain were known to have an impact on the physicochemical properties and biological activity [86].

The antifungal activity and surface activity was enhanced in the order straight $(\mathrm{n})>$ iso $>$ anteiso [86,
87]. The $C_{14}$ surfactin was found to have higher foaming capacity in comparison to $C_{13}$ and $C_{15}$ surfactins $[45,88]$. It was also found that a linearized synthetic $\mathrm{C}_{14}$ surfactin showed none or less hemolytic behavior in comparison to cyclic natural $\mathrm{C}_{14}$ surfactin [89]. The fatty acid composition of surfactin has a greater effect on the surface activity than on the surfactin production. Iso-odd fatty acid isomers have higher oil displacement than n-even fatty acid isomers [27]. Our research also proved if the surfactin contained more proportion of $\mathrm{C}_{15}$, the surfactin showed a better oil-washing efficiency and oil displacement efficiency [16]. Therefore, in the study of the structural diversity of surfactin, the directional modification of the fatty acid chain of the surfactin product is of great significance for the application of surfactin.

As illustrated in Fig. 2, branched-chain amino acids are closely related to the fatty acyl moiety of surfactin. Hence, amino acids added to the culture medium affect not only the amino acid moiety in the peptide ring but also the hydroxyl fatty acid moiety in the produced lipopeptide. For example, adding Arg, Gln or Val to the medium increased the proportion of surfactin with even $\beta$-hydroxy fatty acid components $\mathrm{C}_{14}$ and $\mathrm{C}_{16}$, whereas the addition of Cys, His, Ile, Leu, Met, and Ser enhanced the odd $\beta$-hydroxy fatty acids in B. subtilis TD7 [90]. Since then, people began to change the fatty acid composition of the surfactin products by modifying or strengthening the relevant amino acid synthesis pathways. Coutte et al. increased the production of surfactin via intracellular L-leucine overproduction by genetically engineering B. subtilis 168 [35]. Notably, not only the yield of surfactin was maximized, but the structural components of surfactin were also changed after knocking out the global regulatory factor CodY, which inhibits the expression of the ilv-leu operon. The relative proportions of $\mathrm{C}_{13}$ and $\mathrm{C}_{14}$ in the control strain were $39.7 \%$ and $21.2 \%$, respectively, which changed to $26.5 \%$ and $40.6 \%$ after knocking out $\operatorname{cod} Y$. Later, Coutte et al. further studied the branchedchain amino acid metabolic pathway and found that the proportion of surfactin with linear $\mathrm{C}_{14}$ was increased 2.5 times after knocking out the $l p d V$ gene responsible for the final degradation of branched-chain amino acids [34].

Obviously, it is difficult to produce directional fatty acid structures of surfactin by using exogenously added amino acid components or internally engineered amino acid synthesis pathways. In order to purposefully modify the structure of fatty acids, it is necessary to understand how the 3-hydroxy fatty acids participate in the lipoinitiation reaction of surfactin biosynthesis. Kraas et al. revealed the mechanism of lipoinitiation by functional dissection of surfactin synthetase [32]. In the initial step of surfactin biosynthesis, the substrate 3-hydroxy fatty acid is combined with coenzyme A ( $\mathrm{CoA})$ under the control of fatty 
acyl CoA ligases (FACLs), which activates the acylation of the first amino acid Glu in the form of a CoA-activated 3-hydroxy fatty acid. Furthermore, gene deletion studies of four putative fatty acyl CoA ligases (FACLs) showed that the yield of surfactin was reduced by $38-55 \%$ with a single knockout of one gene, while the surfactin yield was about $20-65 \%$ when $2-3$ genes were knocked out, and decrease by $84 \%$ when the four putative fatty acyl CoA ligases were all knocked out. These results showed that fatty acyl CoA ligases have a significant effect on surfactin biosynthesis. It should also be noted that deactivating the four putative FACLs does not result in a complete loss of the ability to synthesize surfactin. It therefore seems that there are still fundamental discoveries to be made, more FACLs need to be found and there are still other pathways providing the fatty acid moiety for surfactin production, for example, through transthiolation from ACPs to CoA.

The pool of fatty acid-S-CoA substrates contains many different CoA-activated 3-hydroxy fatty acids which are not all incorporated into surfactin. The SrfAA starter $\mathrm{C}$-domain can catalyze the direct formation of an amide bond between a fatty acyl-CoA and an amino acid moiety. Thus, the substrate selectivity of the C-domain in the initiation module (C-AGlu-PCP) of surfactin synthetase SrfAA plays a more important role in the fatty acyl structure of surfactin. This will give us new insights for the rational design and genetic modification of the fatty acyl structure of surfactin. The $C$ domain of the NRPS synthesis initiation module srfA-A1 may be an important target for fatty acyl structural modification. Changing the specificity of the starter $\mathrm{C}$-domain for fatty acids or swapping the starter C-domains in the initiation module of surfactin synthetase SrfAA for those from foreign NRPSs is a potential way to generate novel surfactin structures with a specific fatty acyl moiety. The exciting work by Kraas et al. was confirmed by Chooi and Tang, who demonstrated that the starter C-domain of SrfAA synthetase is really a worthwhile research subject [91]. Regrettably, there have been no further reports investigating $\mathrm{C}$-domain modification.

\section{Conclusion and prospects}

Surfactin has attracted considerable attention in research and industrial applications due to its various biological and physico-chemical properties, but the current fermentation processes are cost-prohibitive and cannot meet the needs of industrial applications. Controlling the foaming during the fermentation process remains a challenge in the industrial fermentation of biosurfactants. Loss of product, nutrients, and cells caused by foam overflow needs to be solved through process- and reactor optimization. In addition, finding cheap biomass materials is also an effective means to reduce the cost of fermentation and promote the industrial application of surfactin.

With advances in genetic engineering and synthetic biology, the construction of strains with high yield, high conversion rate and high production rate will become feasible in the near future. Creating novel surfactin compounds with new structures is also the key to promote their broader application. The studies on NRPS modules and combinatorial biosynthesis technology provide the basis for the modification of the surfactin peptide loop. However, there are still many hurdles to overcome, including the functional connection between modules, uncertainty about the effects of the modified peptide products, and the low yields. On the other hand, there are still no technical means that would enable the directed regulation of the fatty acyl structure of surfactin. Enhancing the de-novo synthesis pathway of fatty acid precursors may be a good strategy to increasing the production of surfactin with a specific fatty acyl structure. The substrate specificity of the initiation module (C-AGlu-PCP) needs to be analyzed further.

\section{Abbreviations \\ CLPs: cyclic lipopeptides; EOR: enhanced oil recovery; MEOR: microbial enhancement of oil recovery; FHW: feather hydrolysate waste; GMW: gluta- mate mill waste; NRPS: nonribosomal peptide synthetase; LCFAs: long chain fatty acids; PMF: proton motive force; QS: quorum-sensing; FACLs: fatty acyl CoA ligases; FA-Glu: fatty-acyl-glutamate; CoA: coenzyme A.}

\section{Authors' contributions}

$\mathrm{H}-\mathrm{FX}$ and LS overall coordinated, finished the illustrations, and finalized the manuscript. L-YY participated in revising the draft the manuscript. All authors read and approved the final manuscript.

\section{Acknowledgements}

Not applicable.

Competing interests

The authors declare that they have no competing interests.

\section{Availability of data and materials}

Not applicable. The manuscript contains an overview of the literature and all data are available in it.

\section{Consent for publication}

Not applicable.

Ethics approval and consent to participate

Not applicable.

\section{Funding}

This work was supported by the National Natural Science Foundation of China (No. 21576133) and the Jiangsu Synergetic Innovation Center for Advanced Bio-Manufacture.

\section{Publisher's Note}

Springer Nature remains neutral with regard to jurisdictional claims in published maps and institutional affiliations. 
Received: 14 November 2018 Accepted: 18 February 2019 Published online: 28 February 2019

\section{References}

1. Deravel J, Lemiere S, Coutte F, Krier F, Van Hese N, Bechet M, Sourdeau N Hofte M, Lepretre A, Jacques P. Mycosubtilin and surfactin are efficient, low ecotoxicity molecules for the biocontrol of lettuce downy mildew. Appl Environ Microbiol. 2014;98:6255-64.

2. Zhang J, Xue Q, Gao H, Lai H, Wang P. Production of lipopeptide biosurfactants by Bacillus atrophaeus 5-2a and their potential use in microbial enhanced oil recovery. Microb Cell Fact. 2016;15:168.

3. Chen W-C, Juang R-S, Wei Y-H. Applications of a lipopeptide biosurfactant, surfactin, produced by microorganisms. Biochem Eng J. 2015;103:158-69.

4. Rodrigues L, Banat IM, Teixeira J, Oliveira R. Biosurfactants: potential applications in medicine. J Antimicrob Chemother. 2006;57:609-18.

5. Meena KR, Kanwar SS. Lipopeptides as the antifungal and antibacterial agents: applications in food safety and therapeutics. Biomed Res Int. 2015. https://doi.org/10.1155/2015/473050.

6. Pathak KV, Keharia H, Gupta K, Thakur SS, Balaram P. Lipopeptides from the banyan endophyte, Bacillus subtilis K1: mass spectrometric characterization of a library of fengycins. J Am Soc Mass Spectrom. 2012;23:1716-28.

7. Cochrane SA, Vederas JC. Lipopeptides from Bacillus and Paenibacillus spp.: a gold mine of antibiotic candidates. Med Res Rev. 2016;36:4-31.

8. Xue Y, Wang MY, Zhao PC, Quan CS, Li X, Wang LN, Gao WN, Li JH, Zu XY, Fu DL, et al. Gram-negative bacilli-derived peptide antibiotics developed since 2000. Biotechnol Lett. 2018;40:1271-87.

9. Zhao HB, Shao DY, Jiang CM, Shi JL, Li Q, Huang QS, Rajoka MSR, Yang H, Jin ML. Biological activity of lipopeptides from Bacillus. Appl Microbiol Biotechnol. 2017;101:5951-60.

10. Romano A, Vitullo D, Senatore M, Lima G, Lanzotti V. Antifungal cyclic lipopeptides from Bacillus amyloliquefaciens strain BO5A. J Nat Prod. 2013;76:2019-25

11. Arima K, Kakinuma A, Tamura G. Surfactin, a crystalline peptidelipid surfactant produced by Bacillus subtilis: isolation, characterization and its inhibition of fibrin clot formation. Biochem Biophys Res Commun. 1968:31:488-94.

12. Seydlova G, Svobodova J. Review of surfactin chemical properties and the potential biomedical applications. Cent Eur J Public Health. 2008:3:123-33.

13. Yang $H$, Li X, Li X, Yu H, Shen Z. Identification of lipopeptide isoforms by MALDI-TOF-MS/MS based on the simultaneous purification of iturin, fengycin, and surfactin by RP-HPLC. Anal Bioanal Chem. 2015;407:2529-42.

14. Zou A, Liu J, Garamus VM, Yang Y, Willumeit R, Mu B. Micellization activity of the natural lipopeptide Glu(1), ASP(5) surfactin-C15 in aqueous solution. J Phys Chem B. 2010;114:2712-8.

15. Bordoloi NK, Konwar BK. Microbial surfactant-enhanced mineral oil recovery under laboratory conditions. Colloids Surf B Biointerfaces. 2008:63:73-82.

16. Liu Q, Lin J, Wang W, Huang H, Li S. Production of surfactin isoforms by Bacillus subtilis BS-37 and its applicability to enhanced oil recovery under laboratory conditions. Biochem Eng J. 2015;93:31-7.

17. Amani $\mathrm{H}$, Haghighi M, Keshtkar MJ. Production and optimization of microbial surfactin by Bacillus subtilis for ex situ enhanced oil recovery. Pet Sci Technol. 2013:31:1249-58.

18. Lai C-C, Huang Y-C, Wei Y-H, Chang J-S. Biosurfactant-enhanced removal of total petroleum hydrocarbons from contaminated soil. J Hazard Mater. 2009;167:609-14.

19. Wu Y-S, Ngai S-C, Goh B-H, Chan K-G, Lee L-H, Chuah L-H. Anticancer activities of surfactin and potential application of nanotechnology assisted surfactin delivery. Front Pharmacol. 2017;8:761.

20. Chen C, Lin J, Wang W, Huang H, Li S. Cost-effective production of surfactin from xylose-rich corncob hydrolysate using Bacillus subtilis BS-37. Waste Biomass Valori. 2017. https://doi.org/10.1007/s12649-017-0052-5.

21. Zhi Y, Wu Q, Xu Y. Production of surfactin from waste distillers' grains by co-culture fermentation of two Bacillus amyloliquefaciens strains. Bioresour Technol. 2017;235:96-103.
22. Moya Ramirez I, Altmajer Vaz D, Banat IM, Marchant R, Jurado Alameda E, Garcia Roman M. Hydrolysis of olive mill waste to enhance rhamnolipids and surfactin production. Bioresour Technol. 2016;205:1-6.

23. Wei YH, Lai CC, Chang JS. Using Taguchi experimental design methods to optimize trace element composition for enhanced surfactin production by Bacillus subtilis ATCC 21332. Process Biochem. 2007;42:40-5.

24. Wei YH, Chu IM. Mn ${ }^{2+}$ improves surfactin production by Bacillus subtilis. Biotechnol Lett. 2002;24:479-82.

25. Yi G, Liu O, Lin J, Wang W, Huang H, Li S. Repeated batch fermentation for surfactin production with immobilized Bacillus subtilis BS-37: twostage $\mathrm{pH}$ control and foam fractionation. J Chem Technol Biotechnol. 2017:92:520-5.

26. Kaneda T. Fatty acids of the genus Bacillus: an example of branched-chain preference. Bacteriol Rev. 1977;41:391-418.

27. Youssef NH, Duncan KE, McInerney MJ. Importance of 3-hydroxy fatty acid composition of lipopeptides for biosurfactant activity. Appl Environ Microbiol. 2005;71:7690-5.

28. Ongena M, Jacques P. Bacillus lipopeptides: versatile weapons for plant disease biocontrol. Trends Microbiol. 2008;16:115-25.

29. Mootz HD, Marahiel MA. Biosynthetic systems for nonribosomal peptide antibiotic assembly. Curr Opin Chem Biol. 1997;1:543-51.

30. Yeh E, Kohli RM, Bruner SD, Walsh CT. Type II thioesterase restores activity of a NRPS module stalled with an aminoacyl-S-enzyme that cannot be elongated. ChemBioChem. 2004:5:1290-3.

31. Kopp F, Marahiel MA. Macrocyclization strategies in polyketide and nonribosomal peptide biosynthesis. Nat Prod Rep. 2007;24:735-49.

32. Kraas Fl, Helmetag $\bigvee$, Wittmann M, Strieker M, Marahiel MA. Functional dissection of surfactin synthetase initiation module reveals insights into the mechanism of lipoinitiation. Chem Biol. 2010;17:872-80.

33. Wu Q, Zhi Y, Xu Y. Systematically engineering the biosynthesis of a green biosurfactant surfactin by Bacillus subtilis 168. Metab Eng. 2018:52:87-97.

34. Dhali D, Coutte F, Arias AA, Auger S, Bidnenko V, Chataigne G, Lalk M, Niehren J, de Sousa J, Versari C, Jacques P. Genetic engineering of the branched fatty acid metabolic pathway of Bacillus subtilis for the overproduction of surfactin C-14 isoform. Biotechnol J. 2017;12:10.

35. Coutte F, Niehren J, Dhali D, John M, Versari C, Jacques P. Modeling leucine's metabolic pathway and knockout prediction improving the production of surfactin, a biosurfactant from Bacillus subtilis. Biotechnol J. 2015;10:1216-34.

36. Singh AK, Zhang Y-M, Zhu K, Subramanian C, Li Z, Jayaswal RK, Gatto C, Rock CO, Wilkinson BJ. FabH selectivity for anteiso branched-chain fatty acid precursors in low-temperature adaptation in Listeria monocytogenes. FEMS Microbiol Lett. 2009;301:188-92.

37. Choi KH, Heath RJ, Rock CO. Beta-ketoacyl-acyl carrier protein synthase III $(\mathrm{FabH})$ is a determining factor in branched-chain fatty acid biosynthesis. J Bacteriol. 2000:182:365-70.

38. Nickels JD, Chatterjee S, Mostofian B, Stanley CB, Ohl M, Zolnierczuk P, Schulz R, Myles DAA, Standaert RF, Elkins JG, et al. Bacillus subtilis lipid extract, a branched-chain fatty acid membrane model. J Phys Chem Lett. 2017;8:4214-7.

39. Park JH, Lee KH, Kim TY, Lee SY. Metabolic engineering of Escherichia coli for the production of L-valine based on transcriptome analysis and in silico gene knockout simulation. Proc Natl Acad Sci USA. 2007;104:7797-802

40. Park JH, Lee SY. Metabolic pathways and fermentative production of L-aspartate family amino acids. Biotechnol J. 2010;5:560-77.

41. Debarbouille M, Gardan R, Arnaud M, Rapoport G. Role of BkdR, a transcriptional activator of the SigL-dependent isoleucine and valine degradation pathway in Bacillus subtilis. J Bacteriol. 1999;181:2059-66.

42. Cheng JL, Ma JC, Lin JS, Fan ZC, Cronan JE, Wang HH. Only one of the five Ralstonia solanacearum long-chain 3-ketoacyl-acyl carrier protein synthase homologues functions in fatty acid synthesis. Appl Environ Microbiol. 2012;78:1563-73.

43. Janssen HJ, Steinbuchel A. Fatty acid synthesis in Escherichia coli and its applications towards the production of fatty acid based biofuels. Biotechnol Biofuels. 2014;7:7

44. Lee DS, Yamada A, Sugimoto H, Matsunaga I, Ogura H, Ichihara K, Adachi S, Park SY, Shiro Y. Substrate recognition and molecular mechanism of fatty acid hydroxylation by cytochrome P450 from Bacillus subtiliscrystallographic, spectroscopic, and mutational studies. J Biol Chem. 2003:278:9761-7. 
45. Youssef NH, Wofford N, Mclnerney MJ. Importance of the long-chain fatty acid beta-hydroxylating cytochrome P450 enzyme YbdT for lipopeptide biosynthesis in Bacillus subtilis strain OKB105. Int J Mol Sci. 2011;12:1767-86.

46. Zhi Y, Wu Q, Xu Y. Genome and transcriptome analysis of surfactin biosynthesis in Bacillus amyloliquefaciens MT45. Sci Rep. 2017;7:40976.

47. Zhou D, Hu F, Lin J, Wang W, Li S. Genome and transcriptome analysis of Bacillus velezensis BS-37, an efficient surfactin producer from glycerol, in response to D-/L-leucine. Microbiol Open. 2019. https://doi.org/10.1002/ mbo3.794.

48. Liu X, Ren B, Chen M, Wang H, Kokare CR, Zhou X, Wang J, Dai H, Song F, Liu M, et al. Production and characterization of a group of bioemulsifiers from the marine Bacillus velezensis strain $\mathrm{H} 3$. Appl Environ Microbiol. 2010;87:1881-93.

49. Lee S-J, Pan J-G, Park S-H, Choi S-K. Development of a stationary phasespecific autoinducible expression system in Bacillus subtilis. J Biotechnol. 2010;149:16-20.

50. Cheng J, Guan C, Cui W, Zhou L, Liu Z, Li W, Zhou Z. Enhancement of a high efficient autoinducible expression system in Bacillus subtilis by promoter engineering. Protein Express Purif. 2016;127:81-7.

51. Jiao $S, L i X, Y u H$, Yang H, Li X, Shen Z. In situ enhancement of surfactin biosynthesis in Bacillus subtilis using novel artificial inducible promoters. Biotechnol Bioeng. 2017;114:832-42.

52. Willenbacher J, Mohr T, Henkel M, Gebhard S, Mascher T, Syldatk C, Hausmann R. Substitution of the native srfA promoter by constitutive P-veg in two B. subtilis strains and evaluation of the effect on surfactin production. J Biotechnol. 2016;224:14-7.

53. Sun H, Bie X, Lu F, Lu Y, Wu Y, Lu Z. Enhancement of surfactin production of Bacillus subtilis fmbR by replacement of the native promoter with the Pspac promoter. Can J Microbiol. 2009;55:1003-6.

54. Coutte F, Leclere V, Bechet M, Guez JS, Lecouturier D, Chollet-Imbert M, Dhulster P, Jacques P. Effect of pps disruption and constitutive expression of srfA on surfactin productivity, spreading and antagonistic properties of Bacillus subtilis 168 derivatives. J Appl Microbiol. 2010;109:480-91.

55. Deleu M, Bouffioux O, Razafindralambo H, Paquot M, Hbid C, Thonart $P$, Jacques P, Brasseur R. Interaction of surfactin with membranes: a computational approach. Langmuir. 2003;19:3377-85.

56. Deleu M, Lorent J, Lins L, Brasseur R, Braun N, El Kirat K, Nylander T, Dufrene YF, Mingeot-Leclercq M-P. Effects of surfactin on membrane models displaying lipid phase separation. BBA-Biomembranes. 2013;1828:801-15.

57. Li X, Yang H, Zhang D, Li X, Yu H, Shen Z. Overexpression of specific proton motive force-dependent transporters facilitate the export of surfactin in Bacillus subtilis. J Ind Microbiol Biotechnol. 2015;42:93-103.

58. Tsuge K, Ohata Y, Shoda M. Gene yerP, involved in surfactin self-resistance in Bacillus subtilis. Antimicrob Agents Chemother. 2001;45:3566-73.

59. Lomovskaya O, Zgurskaya HI, Totrov M, Watkins WJ. Waltzing transporters and 'the dance macabre' between humans and bacteria. Nat Rev Drug Discov. 2007;6:56-65.

60. Pottathil M, Jung A, Lazazzera BA. CSF, a species-specific extracellular signaling peptide for communication among strains of Bacillus subtilis and Bacillus mojavensis. J Bacteriol. 2008;190:4095-9.

61. Shank EA, Kolter R. Extracellular signaling and multicellularity in Bacillus subtilis. Curr Opin Microbiol. 2011;14:741-7.

62. Stefanic P, Decorosi F, Viti C, Petito J, Cohan FM, Mandic-Mulec I. The quorum sensing diversity within and between ecotypes of Bacillus subtilis. Environ Microbiol. 2012;14:1378-89.

63. Cosby WM, Vollenbroich D, Lee OH, Zuber P. Altered srf expression in Bacillus subtilis resulting from changes in culture $\mathrm{pH}$ is dependent on the SpoOK oligopeptide permease and the ComQX system of extracellular control. J Bacteriol. 1998;180:1438-45.

64. Guan C, Cui W, Cheng J, Zhou L, Guo J, Hu X, Xiao G, Zhou Z. Construction and development of an auto-regulatory gene expression system in Bacillus subtilis. Microb Cell Fact. 2015;14:150. https://doi.org/10.1186/s1293 4-015-0341-2.

65. Marahiel MA, Nakano MM, Zuber P. Regulation of peptide antibiotic production in Bacillus. Mol Microbiol. 1993;7:631-6.
66. Lazazzera BA, Solomon JM, Grossman AD. An exported peptide functions intracellularly to contribute to cell density signaling in B. subtilis. Cell. 1997:89:917-25.

67. Auchtung JM, Lee CA, Grossman AD. Modulation of the ComA-dependent quorum response in Bacillus subtilis by multiple rap proteins and $\mathrm{Phr}$ peptides. J Bacteriol. 2006;188:5273-85.

68. Ohsawa T, Tsukahara K, Sato T, Ogura M. Superoxide stress decreases expression of srfA through inhibition of transcription of the comQXP quorum-sensing locus in Bacillus subtilis. J Biochem. 2006;139:203-11.

69. Jung J, Yu KO, Ramzi AB, Choe SH, Kim SW, Han SO. Improvement of surfactin production in Bacillus subtilis using synthetic wastewater by overexpression of specific extracellular signaling peptides, comX and phrC. Biotechnol Bioeng. 2012;109:2349-56.

70. Serror P, Sonenshein AL. CodY is required for nutritional repression of Bacillus subtilis genetic competence. J Bacteriol. 1996;178:5910-5.

71. Chumsakul O, Takahashi H, Oshima T, Hishimoto T, Kanaya S, Ogasawara $\mathrm{N}$, Ishikawa S. Genome-wide binding profiles of the Bacillus subtilis transition state regulator $\mathrm{AbrB}$ and its homolog Abh reveals their interactive role in transcriptional regulation. Nucleic Acids Res. 2011;39:414-28.

72. Zhang Y, Nakano S, Choi SY, Zuber P. Mutational analysis of the Bacillus subtilis RNA polymerase at C-terminal domain supports the interference model of Spx-dependent repression. J Bacteriol. 2006;188:4300-11.

73. Lopez D, Vlamakis H, Losick R, Kolter R. Paracrine signaling in a bacterium. Genes Dev. 2009;23:1631-8.

74. Hayashi K, Ohsawa T, Kobayashi K, Ogasawara N, Ogura M. The $\mathrm{H}_{2} \mathrm{O}_{2}$ stress-responsive regulator PerR positively regulates srfA expression in Bacillus subtilis. J Bacteriol. 2005;187:6659-67.

75. Ruckert C, Blom J, Chen XH, Reva O, Borriss R. Genome sequence of $B$. amyloliquefaciens type strain DSM7(T) reveals differences to plant-associated B. amyloliquefaciens FZB42. J Biotechnol. 2011;155:78-85.

76. Baltz RH. Combinatorial biosynthesis of cyclic lipopeptide antibiotics: a model for synthetic biology to accelerate the evolution of secondary metabolite biosynthetic pathways. ACS Synth Biol. 2014;3:748-58.

77. Stachelhaus T, Schneider A, Marahiel MA. Rational design of peptide antibiotics by targeted replacement of bacterial and fungal domains. Science. 1995;269:69-72.

78. Stachelhaus T, Schneider A, Marahiel MA. Engineered biosynthesis of peptide antibiotics. Biochem Pharmacol. 1996;52:177-86.

79. Schneider A, Stachelhaus T, Marahiel MA. Targeted alteration of the substrate specificity of peptide synthetases by rational module swapping. Mol Gen Genet. 1998;257:308-18.

80. Eppelmann K, Stachelhaus T, Marahiel MA. Exploitation of the selectivityconferring code of nonribosomal peptide synthetases for the rational design of novel peptide antibiotics. Biochemistry. 2002;41:9718-26.

81. Kries H, Wachtel R, Pabst A, Wanner B, Niquille D, Hilvert D. Reprogramming nonribosomal peptide synthetases for "clickable" amino acids. Angew Chem Int Ed Engl. 2014;53:10105-8.

82. Mootz HD, Kessler N, Linne U, Eppelmann K, Schwarzer D, Marahiel MA. Decreasing the ring size of a cyclic nonribosomal peptide antibiotic by in-frame module deletion in the biosynthetic genes. J Am Chen Soc. 2002;124:10980-1.

83. Jiang J, Gao L, Bie X, Lu Z, Liu H, Zhang C, Lu F, Zhao H. Identification of novel surfactin derivatives from NRPS modification of Bacillus subtilis and its antifungal activity against Fusarium moniliforme. BMC Microbiol. 2016;16:31.

84. Marti ME, Colonna WJ, Reznik G, Pynn M, Jarrell K, Lamsal B, Glatz CE. Production of fatty-acyl-glutamate biosurfactant by Bacillus subtilis on soybean co-products. Biochem Eng J. 2015;95:48-55.

85. Reznik GO, Vishwanath P, Pynn MA, Sitnik JM, Todd JJ, Wu J, Jiang Y, Keenan BG, Castle AB, Haskell RF, et al. Use of sustainable chemistry to produce an acyl amino acid surfactant. Appl Microbiol Biotechnol. 2010;86:1387-97.

86. Ahimou F, Jacques P, Deleu M. Surfactin and iturin A effects on Bacillus subtilis surface hydrophobicity. Enzyme Microb Technol. 2000;27:749-54.

87. Bechet M, Castera-Guy J, Guez J-S, Chihib N-E, Coucheney F, Coutte F, Fickers $P$, Leclere $V$, Wathelet $B$, Jacques P. Production of a novel mixture of mycosubtilins by mutants of Bacillus subtilis. Bioresour Technol. 2013;145:264-70. 
88. Fickers P, Guez J-S, Damblon C, Leclere V, Bechet M, Jacques P, Joris B. High-level biosynthesis of the anteiso- $\mathrm{C}-17$ isoform of the antibiotic mycosubtilin in Bacillus subtilis and characterization of its candidacidal activity. Appl Environ Microbiol. 2009;75:4636-40.

89. Dufour S, Deleu M, Nott K, Wathelet B, Thonart P, Paquot M. Hemolytic activity of new linear surfactin analogs in relation to their physico-chemical properties. BBA-Gen Subjects. 2005;1726:87-95.
90. Liu J-F, Yang J, Yang S-Z, Ye R-Q, Mu B-Z. Effects of different amino acids in culture media on surfactin variants produced by Bacillus subtilis TD7. Appl Biochem Biotechnol. 2012;166:2091-100.

91. Chooi Y-H, Tang Y. Adding the lipo to lipopeptides: do more with less. Chem Biol. 2010;17:791-3.
Ready to submit your research? Choose BMC and benefit from:

- fast, convenient online submission

- thorough peer review by experienced researchers in your field

- rapid publication on acceptance

- support for research data, including large and complex data types

- gold Open Access which fosters wider collaboration and increased citations

- maximum visibility for your research: over $100 \mathrm{M}$ website views per year

At BMC, research is always in progress.

Learn more biomedcentral.com/submissions 